\title{
An Exactly Soluble Multiatom-Multiphoton Coupling Model
}

\author{
A. N. F. Aleixo, \\ Instituto de Física, Universidade Federal do Rio de Janeiro, Rio de Janeiro, RJ Brazil
}

A. B. Balantekin,

Department of Physics, University of Wisconsin, Madison, Wisconsin 53706 USA

and M. A. Cândido Ribeiro

Departamento de Física, Instituto de Biociências, Letras e Ciências Exatas, UNESP, São José do Rio Preto, SP, Brazil

Received on 27 May, 2003

\begin{abstract}
A class of bound-state problems which represent the interaction between a system of $N$ two-level atoms and an electromagnetic radiation via an $m$-photon process is studied, with $m=1,2,3, \ldots$ We also evaluate some nonlinear effects usually related with the polarisability of the medium and with the dependence on the intensity of the radiation field on the matter-radiation interaction. We obtain exact values for the eigenstates and eigenvalues for all values of total angular momentum of the system and all possible number $m$ of photons involved in the interaction. We give explicit analytic expressions for small numbers of atoms and discuss some aspects of the composition of the eigenstates and of the spectra obtained in these cases.
\end{abstract}

\section{Introduction}

A standard model most used in quantum optics idealizes the interaction of the matter with the radiation by a simple Hamiltonian of a two-level atom coupled to a single bosonic mode [1]. This Hamiltonian is a central ingredient in the quantized description of any optical system involving the interaction between light and atoms. For this Hamiltonian, mostly the single-particle situation has been studied [2-13]. The purpose of this paper is to give the exact and complete solution for this model when we consider the presence of $N$ two-level systems which interact with a single-mode radiation via a single or multi-photon process, that take place when the energy separation between the atomic levels is close to the energy of $m$ radiation quanta. In this case the effects of the spatial distribution of the particles it is not taken into account and the spin angular momentum $\hat{\mathbf{S}}_{i}$ of each particle contributes to form a total angular momentum $\hat{\mathbf{J}}$ of the system. We consider in this study the effects due to the nonlinear radiation polarisability with the inclusion of a nonlinear term in the Hamiltonian [8]. This kind of nonlinear effect is usually considered in the study of micromasers.

Another nonlinear extension of our study takes the coupling between matter and the radiation to depend on the intensity of the radiation field [4-6,9]. It is interesting since this kind of interaction means that effectively the coupling is proportional to the amplitude of the field, corresponding to a more realistic physical situation. We obtain exact values for the eigenstates and eigenvalues for all values of $j$ and for any number $m$ of photon involved in the atom-radiation interaction. We give explicit analytic expressions for small numbers of atoms and discuss some aspects of the spectra obtained in these cases.

This paper is organized in the following way, in Section II we introduce the multiatom Hamiltonian with multiphoton interaction and its solution; in Section III we do the same for an intensity-dependent radiation interaction; application are presented in Section IV and some numerical results with its discussion are presented in section V. Finally, conclusion and brief remarks close the paper in Section VI.

\section{Multi-atom model with multi- photon interaction}

The Hamiltonian describing the interaction between a single-mode radiation and $N$ two-level systems via an $m$ photon process, when we include nonlinear effects, can be written as

$\hat{\mathbf{H}}=\hat{\mathbf{H}}^{(0)}+\hat{\mathbf{H}}^{(1)}$, where $\left\{\begin{array}{l}\hat{\mathbf{H}}^{(0)}=\hbar \omega \hat{a}^{\dagger} \hat{a}+m \hbar \omega \hat{\mathbf{J}}_{3} \\ \hat{\mathbf{H}}^{(1)}=\hat{\mathbf{H}}_{\mathrm{int}}+\hat{\mathbf{k}}-\hbar \Delta_{m} \hat{\mathbf{J}}_{3}\end{array}\right.$

with $m=1,2,3, \ldots$,

$$
\hat{\mathbf{H}}_{\mathrm{int}}=\hbar \Omega\left(\hat{a}^{m} \hat{\mathbf{J}}_{+}+\hat{a}^{\dagger m} \hat{\mathbf{J}}_{-}\right) \text {and } \hat{\mathbf{k}}=\hbar \Theta \hat{a}^{\dagger 2} \hat{a}^{2} \text {. }
$$

Here $\Omega$ is the matter-radiation interaction parameter, $\Theta$ is the nonlinear medium parameter, related to the third-order nonlinear susceptibility, $\Delta_{m}=m \omega-\omega_{0}$ is a constant related to the detuning of the system and $\omega\left(\omega_{0}\right)$ is the field (atomic transition) frequency. In the above expressions, 


$$
\hat{\mathbf{J}}_{ \pm}=\hat{\mathbf{J}}_{1} \pm i \hat{\mathbf{J}}_{2},
$$

where $\hat{\mathbf{J}}_{i}$ (with $i=1,2$, and 3 ) are the components of the total angular momentum of the system, obtained by the sum of the spin angular momentum of the $N$ two-level atoms.

To obtain the eigenstates and eigenvalues for the Hamiltonian (2.1) we first remember that the bosonic operators, when applied to the number states, satisfy the following properties

$$
\hat{a}|n\rangle=\sqrt{n}|n-1\rangle, \text { and } \hat{a}^{\dagger}|n\rangle=\sqrt{n+1}|n+1\rangle,
$$

where the excited bosonic states are written as

$$
|n\rangle=\frac{\left(\hat{a}^{\dagger}\right)^{n}}{\sqrt{n !}}|0\rangle .
$$

Now, if we introduce the operators

$$
\hat{\mathcal{A}}_{m}=\frac{\hat{a}^{m}}{m}, \quad \hat{\mathcal{A}}_{m}^{\dagger}=\frac{\hat{a}^{\dagger m}}{m},
$$

and

$$
\hat{\mathcal{N}}_{m}=\frac{1}{2 m}\left(\hat{a}^{\dagger} \hat{a}+\hat{a} \hat{a}^{\dagger}\right),
$$

that satisfy the following commutation relations

$$
\left[\hat{\mathcal{N}}_{m}, \hat{\mathcal{A}}_{m}\right]=-\hat{\mathcal{A}}_{m}, \quad\left[\hat{\mathcal{N}}_{m}, \hat{\mathcal{A}}_{m}^{\dagger}\right]=\hat{\mathcal{A}}_{m}^{\dagger},
$$

and

$$
\left[\hat{\mathcal{A}}_{m}, \hat{\mathcal{A}}_{m}^{\dagger}\right]=\frac{1}{m^{2}}\left\{\frac{(\hat{n}+m) !}{\hat{n} !}-\frac{\hat{n} !}{(\hat{n}-m) !}\right\},
$$

where

$$
\hat{n}=\hat{a}^{\dagger} \hat{a}
$$

then we can rewrite the components of the total Hamiltonian (2.1) as

$$
\left\{\begin{array}{l}
\hat{\mathbf{H}}^{(0)}=m \hbar \omega\left(\hat{\mathcal{N}}_{m}+\hat{\mathbf{J}}_{3}-\frac{1}{2 m}\right), \\
\hat{\mathbf{H}}_{\text {int }}=m \hbar \Omega\left(\hat{\mathcal{A}}_{m} \hat{\mathbf{J}}_{+}+\hat{\mathcal{A}}_{m}^{\dagger} \hat{\mathbf{J}}_{-}\right), \\
\hat{\mathbf{k}}=m^{2} \hbar \Theta\left(\hat{\mathcal{N}}_{m}-\frac{1}{2 m}\right)\left(\hat{\mathcal{N}}_{m}-\frac{3}{2 m}\right) .
\end{array}\right.
$$

Note that for $m=1$ the new operators reproduce the standard bosonic commutation relations $[\hat{n}, \hat{a}]=-\hat{a},\left[\hat{n}, \hat{a}^{\dagger}\right]=$ $\hat{a}^{\dagger},\left[\hat{a}, \hat{a}^{\dagger}\right]=1$, characteristic of the Heisenberg-Weyl algebra, and for $m=2$ these commutation relations, which can be written as $\left[\hat{\mathcal{N}}_{2}, \hat{\mathcal{A}}_{2}\right]=-\hat{\mathcal{A}}_{2},\left[\hat{\mathcal{N}}_{2}, \hat{\mathcal{A}}_{2}^{\dagger}\right]=\hat{\mathcal{A}}_{2}^{\dagger}$, and $\left[\hat{\mathcal{A}}_{2}, \hat{\mathcal{A}}_{2}^{\dagger}\right]=2 \hat{\mathcal{N}}_{2}$, represent the bosonic realization of the $\mathrm{SU}(1,1)$ algebra.

With the new form (2.9) of the total Hamiltonian elements it is easy to show that the two terms in the Hamiltonian (2.1) commute with each other, $\left[\hat{\mathbf{H}}^{(0)}, \hat{\mathbf{H}}^{(1)}\right]=0$, therefore it is possible to find a common set of eigenstates for them. The simplest way to determine the eigenstates and eigenvalues of that Hamiltonian is by calculating the eigenstates and eigenvalues of $\hat{\mathbf{H}}^{(1)}$. Taking into account that the $\hat{\mathbf{J}}_{i}$ operators can be represented by $(2 j+1) \times(2 j+1)$ matrices, then by using the form of these matrices we can write the operator $\hat{\mathbf{H}}^{(1)}$ as a tridiagonal matrix with these same dimensions of $\hat{\mathbf{J}}_{i}$ and whose elements can be written as

$$
\hat{\mathrm{H}}_{i k}^{(1)}=\left\{\begin{array}{lr}
\hbar \Delta_{m}(i-j-1)+\hat{\mathbf{k}}, & \text { if } \quad k=i \\
\hbar m \Omega \sqrt{(k-1)(2 j-i+1)} \hat{\mathcal{A}}_{m}, & \text { if } \quad k=i+1
\end{array}\right.
$$

with the property $\hat{\mathrm{H}}_{k i}^{(q)}=\left(\hat{\mathrm{H}}_{i k}^{(q)}\right)^{\dagger}$. By considering the properties (2.4) of the bosonic operators and the matrix form of the operator $\hat{\mathbf{H}}^{(1)}$, we introduce the following vector for its eigenstates

$$
\left|\Psi_{n m}\right\rangle=\left[\begin{array}{c}
C_{n}|n\rangle \\
C_{n+m}|n+m\rangle \\
C_{n+2 m}|n+2 m\rangle \\
\vdots \\
C_{n+2 j m}|n+2 j m\rangle,
\end{array}\right] \quad n=0,1,2,3, \cdots
$$

where the $C$ coefficients are to be determined by the eigenvalue equation

$$
\hat{\mathbf{H}}^{(1)}\left|\Psi_{n m}\right\rangle=\mathcal{E}_{n m}\left|\Psi_{n m}\right\rangle
$$

which also determines the eigenvalues $\mathcal{E}_{n m}$. Basically the vector $\left|\Psi_{n m}\right\rangle$ corresponds to the state of the system with total angular momentum $J^{2}=j(j+1) \hbar^{2}$ and each vector component represents one of $2 j+1$ possible values of $J_{z}$. In other words, the component $|n+k m\rangle$ of the vector state $\left|\Psi_{n m}\right\rangle$ represents the quantum state $\left\{n, j, m_{j}=j-k\right\}$. By considering that $\hat{\mathcal{A}}_{m}|k\rangle=0$ if $k<m$ and 


$$
\hat{\mathcal{A}}_{m}|k\rangle=\frac{1}{m} \sqrt{\frac{k !}{(k-m) !}}|k-m\rangle, \quad \text { if } \quad k \geq m,
$$

and also

$$
\hat{\mathcal{A}}_{m}^{\dagger}|k\rangle=\frac{1}{m} \sqrt{\frac{(k+m) !}{k !}}|k+m\rangle, \quad \hat{\mathcal{N}}_{m}^{\dagger}|k\rangle=\frac{1}{m}\left(k+\frac{1}{2}\right)|k\rangle,
$$

then, by substituting Eqs. (2.10) and (2.11) into Eq. (2.12) we obtain the set of $(2 j+1)$ coupled algebraic equations which can be written in a compact form as

$$
\left(1-\delta_{k, 1}\right) \mathrm{h}_{(k-1) k} C_{n+(k-2) m}+\mathrm{h}_{k k} C_{n+(k-1) m}+\left(1-\delta_{k, 2 j+1}\right) \mathrm{h}_{k(k+1)} C_{n+k m}=\mathcal{E}_{n m} C_{n+(k-1) m},
$$

where $k=1,2,3, \ldots, 2 j+1$ and the h-coefficients are defined as

$$
\mathrm{h}_{i k}=\hbar \begin{cases}\Theta[n+(k-1) m][n+(k-1) m-1]-\Delta_{m}(j-k+1), & \text { if } \quad i=k \\ \Omega \sqrt{(k-1)(2 j-k+2)[n+(k-1) m] ! /[n+(k-2) m] !}, & \text { if } i=k-1 .\end{cases}
$$

With this set of $(2 j+1)$ equations plus the normalization condition for the eigenstate it is possible to obtain the $(2 j+2)$ variables that appear in (2.15). This set of equations can be written in a matrix form as

$$
\left[\mathbf{h}-\mathcal{E}_{n m} \mathbf{I}\right] \mathbf{C}=0
$$

where the tridiagonal $\mathbf{h}$-matrix has its elements given by Eq. (2.16) with the symmetry condition $\mathrm{h}_{k i}=\mathrm{h}_{i k}$ and being $\mathbf{C}$ the column matrix

$$
\mathbf{C}=\left[\begin{array}{l}
C_{n} \\
C_{n+m} \\
C_{n+2 m} \\
\vdots \\
C_{n+2 j m} \cdot
\end{array}\right]
$$

The secular equation associated to Eq. (2.17) gives us the algebraic equation to calculate the $2 j+1$ possible values for the eigenvalues $\mathcal{E}_{n m}$

$$
\mathcal{E}_{n m}^{2 j+1}+D_{2 j} \mathcal{E}_{n m}^{2 j}+D_{2 j-1} \mathcal{E}_{n m}^{2 j-1}+\cdots+D_{2} \mathcal{E}_{n m}^{2}+D_{1} \mathcal{E}_{n m}+D_{0}=0,
$$

where the $D$ coefficients are related to the h-matrix elements. Finally, by using Eqs. (2.1), (2.11) and (2.12) we have

$$
\hat{\mathbf{H}}\left|\Psi_{n m}\right\rangle=E_{n m}\left|\Psi_{n m}\right\rangle, \quad \text { where } \quad E_{n m}=(n+j m) \hbar \omega+\mathcal{E}_{n m} .
$$

So far we have only discussed about the possibility of having $n \geq 0$. By considering that $n$ corresponds to the smallest number of photons we might measure in the vector state $\left|\Psi_{n m}\right\rangle$, then when $n \geq 0$, it means that we can find all values of $m_{j}$ from $-j$ to $j$. Therefore when we have
$-2 j \leq n<0$ the vector state $\left|\Psi_{n m}\right\rangle$ corresponds to states in which there are not enough photons to excite the higher $m_{j}$ values even if all of them are absorbed. In this case, the vector state (2.11) that represents this situation will be written as

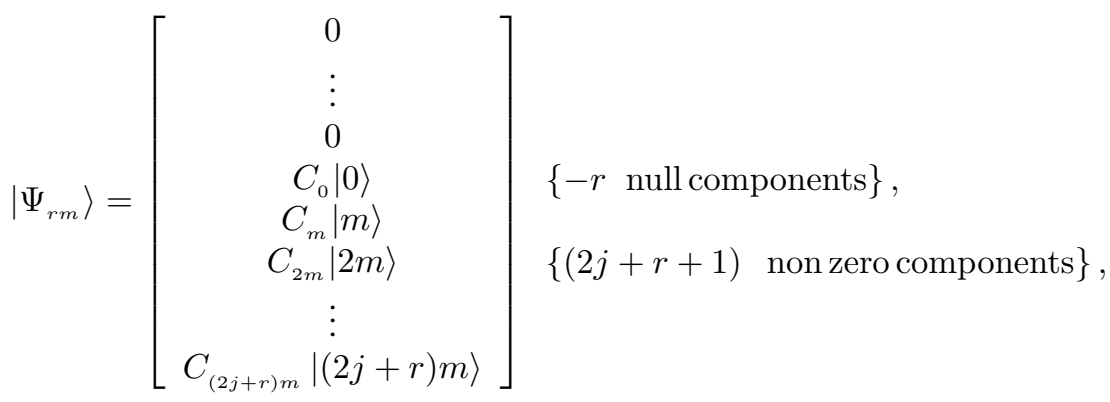


where $-2 j \leq r<0$. In particular, the case $r=-2 j$, that corresponds to the vacuum combined with all particles in their lowest state, represents the ground state of the system. The determination of the eigenstates and eigenvalues of the Hamiltonian (2.1) for these $2 j$ possible $n$ negative values corresponds to a particular application of the general treatment presented before and it will be studied and analyzed for each particular $j$ value in the applications below.

\section{Multi-atom model with intensity- dependent multi-photon interac- tion}

We now introduce an intensity-dependent interaction between matter, constituted by $N$ two-level particles, and radiation via an $m$-photon process by using the following form for the interaction Hamiltonian

$$
\hat{\mathbf{H}}_{\mathrm{int}}=m \hbar \Omega\left(\hat{\mathcal{B}}_{m} \hat{\mathbf{J}}_{+}+\hat{\mathcal{B}}_{m}^{\dagger} \hat{\mathbf{J}}_{-}\right)
$$

where the new operators are defined as

$$
\hat{\mathcal{B}}_{m}=\hat{\mathcal{A}}_{m} \sqrt{\left(m \hat{\mathcal{N}}_{m}-\frac{1}{2}\right)}
$$

and

$$
\hat{\mathcal{B}}_{m}^{\dagger}=\sqrt{\left(m \hat{\mathcal{N}}_{m}-\frac{1}{2}\right)} \hat{\mathcal{A}}_{m}^{\dagger} .
$$

The other terms in the total Hamiltonian (2.1) keep the same forms as used in the previous Section. These facts imply that the diagonal elements of the total Hamiltonian $\hat{\mathbf{H}}$ are still given by Eq. (2.10) and the non diagonal matrix elements now are written as

$$
\hat{\mathrm{H}}_{i k} \equiv \hat{\mathrm{H}}_{i k}^{(1)}=\hbar m \Omega \sqrt{(k-1)(2 j-i+1)} \hat{\mathcal{B}}_{m}, \quad \text { if } \quad k=i+1
$$

with $i, k=1,2,3, \ldots, 2 j+1$, and with the property $\hat{\mathrm{H}}_{k i}=\hat{\mathrm{H}}_{i k}^{\dagger}$. Again, those both terms, $\hat{\mathbf{H}}_{0}$ and $\hat{\mathbf{H}}_{1}$, in the total Hamiltonian (2.1) commute with each other. Therefore, it is possible to use the same technique as used in the preceding Section to obtain the eigenstates and eigenvalues for the total Hamiltonian. Now, by considering that $\hat{\mathcal{B}}_{m}|k\rangle=0$ if $k<m$ and

$$
\hat{\mathcal{B}}_{m}|k\rangle=\frac{1}{m} \sqrt{\frac{k k !}{(k-m) !}}|k-m\rangle=\sqrt{k} \hat{\mathcal{A}}_{m}|k\rangle, \quad \text { if } \quad k \geq m,
$$

and also

$$
\hat{\mathcal{B}}_{m}^{\dagger}|k\rangle=\frac{1}{m} \sqrt{\frac{(k+m)(k+m) !}{k !}}|k+m\rangle=\sqrt{k+m} \hat{\mathcal{A}}_{m}^{\dagger}|k\rangle,
$$

then using Eqs. (3.3) and (2.11) into Eq. (2.12) we obtain a new set of $(2 j+1)$ coupled algebraic equations which can be written in a compact form as

$$
\left(1-\delta_{k, 1}\right) \tilde{\mathrm{h}}_{(k-1) k} C_{n+(k-2) m}+\mathrm{h}_{k k} C_{n+(k-1) m}+\left(1-\delta_{k, 2 j+1}\right) \tilde{\mathrm{h}}_{k(k+1)} C_{n+k m}=\mathcal{E}_{n m} C_{n+(k-1) m},
$$

where $k=1,2,3, \ldots, 2 j+1$ and the new $\tilde{\mathrm{h}}_{i k}$ coefficients, with $i \neq k$, are related with the previous one $\left(\mathrm{h}_{i k}\right)$ by

$$
\tilde{\mathrm{h}}_{i k}=\sqrt{n+(k-1) m} \mathrm{~h}_{i k} \quad \text { with } \quad i=k-1,
$$

and the symmetry property $\tilde{\mathrm{h}}_{i k}=\tilde{\mathrm{h}}_{k i}$. The diagonal $\mathrm{h}_{k k}$ coefficients remain given by Eq. (2.16). Again, the above set of equations can be written in a matrix form as given by Eq. (2.17), except that the non diagonal matrix elements of $\mathbf{h}$ appear now substituted by these $\tilde{\mathrm{h}}_{i k}$ new elements. In this case, to have the spectra we need to resolve Eq. (2.19), where the $D$ coefficients take into account the changes in Eq. (3.6).

It is important to note that the inclusion of negative val- ues of $n$ for this case can be done in the same way as indicated in the previous Section. However, in contrast with what we have for the previous case, the effects of the intensity dependence on the eigenstates and eigenvalues for $n$ negative values will only be observed for $j \geq 1$.

\section{Applications}

\section{A. Single-particle system}

In this case $\mathbf{j}=\mathbf{1} / \mathbf{2}$ and the Hamiltonian $\hat{\mathbf{H}}^{(1)}$ will be a $2 \times 2$ matrix. Applying this Hamiltonian to the eigenstate 
equation (2.12), we obtain the matrix equation (2.17) with

$$
\hat{\mathbf{h}}=\left[\begin{array}{ll}
\mathrm{h}_{11} & \mathrm{~h}_{12} \\
\mathrm{~h}_{12} & \mathrm{~h}_{22}
\end{array}\right]
$$

being the $\mathrm{h}_{i k}$ coefficients given by Eq. (2.16). Then the system of equations (2.15) becomes

$$
\left\{\begin{array}{l}
\mathrm{h}_{11} C_{n}+\mathrm{h}_{12} C_{n+m}=\mathcal{E}_{n m} C_{n} \\
\mathrm{~h}_{12} C_{n}+\mathrm{h}_{22} C_{n+m}=\mathcal{E}_{n m} C_{n+m}^{n}
\end{array}\right.
$$

and, from Eq. (2.19), we have an algebraic quadratic equation with coefficients $D_{1}=-\left(\mathrm{h}_{11}+\mathrm{h}_{22}\right)$ and $D_{0}=$ $h_{11} h_{22}-h_{12}^{2}$ for the eigenvalues which will be given by

$$
\mathcal{E}_{n m}^{( \pm)}=\frac{\hbar}{2}\left\{\Theta\left[2 n^{2}+(2 n+m)(m-1)\right] \pm \sqrt{\frac{4 \Omega^{2}(n+m) !}{n !}+\left[\Theta m(2 n+m-1)+\Delta_{m}\right]^{2}}\right\} .
$$

From Eqs. (4.3), (4.2) and the eigenstate normalization condition we can determine the $C$ coefficients and show that the eigenstates for the total Hamiltonian $\hat{\mathbf{H}}$ can be written as

$$
\left|\Psi_{n m}^{( \pm)}\right\rangle=\frac{1}{\sqrt{1+\left(\gamma_{n+m}^{( \pm)}\right)^{2}}}\left[ \pm \gamma_{n+m}^{( \pm)}|n+m\rangle\right]
$$

with $n=0,1,2, \ldots, m=1,2,3, \ldots$, where

$$
\gamma_{n+m}^{( \pm)}=\frac{1}{\sqrt{1+\delta_{n+m}^{2}} \mp \delta_{n+m}} \quad \text { and } \quad \delta_{n+m}=\frac{\Theta m(2 n+m-1)+\Delta_{m}}{2 \Omega \sqrt{(n+m) ! / n !}}
$$

with eigenvalues given by

$$
E_{n m}^{( \pm)}=\hbar \omega\left(n+\frac{m}{2}\right)+\mathcal{E}_{n m}^{( \pm)}
$$

For the case of intensity-dependent interaction, we obtain (with the change $\mathrm{h}_{i k} \rightarrow \tilde{\mathrm{h}}_{i k}, i \neq k$ ), the following eigenvalues of $\hat{\mathbf{H}}^{(1)}$

$$
\begin{aligned}
\mathcal{E}_{n m}^{( \pm)}=\frac{1}{2} \hbar \Theta\left[2 n^{2}+(2 n+m)(m\right. & -1)] \\
& \pm \frac{1}{2} \hbar \sqrt{\frac{4 \Omega^{2}(n+m)(n+m) !}{n !}+\left[\Theta m(2 n+m-1)+\Delta_{m}\right]^{2}}
\end{aligned}
$$

while the eigenstates for the total Hamiltonian $\hat{\mathbf{H}}$ can be written in the same form given by Esq. (4.4) and (4.5), but with

$$
\delta_{n+m}=\frac{\Theta m(2 n+m-1)+\Delta_{m}}{2 \Omega \sqrt{(n+m)(n+m) ! / n !}} .
$$

The eigenvalues for the total intensity-dependent Hamiltonian will be written as in Eq. (4.6) but, now, with $\mathcal{E}_{n m}^{( \pm)}$given by Eq. (4.7). These results can be reduced to the standard model and to the intensity dependent interaction standard model results $[13,14]$ when we assume the value $m=1$ and turn off the nonlinearity term taking $\Theta=0$.

For $j=1 / 2$ the only possible negative $n$ value is $r=-2 j=-1$ that corresponds to the ground state of the system. Considering this fact and using Eqs. (2.21), (4.1) and (4.2) it is easy to show that

$$
\begin{gathered}
\left|\Psi_{-1 m}\right\rangle=\left[\begin{array}{c}
0 \\
|0\rangle
\end{array}\right], \\
E_{-1 m}=\hbar\left[(m-1) \omega-\frac{\omega_{0}}{2}\right] .
\end{gathered}
$$

In the case of the intensity dependent interaction we have these same results for the eigenvalues and eigenstates as written in Eqs. (4.9).

\section{B. Three-particle system}

In this case we can have $\mathbf{j}=\mathbf{1} / \mathbf{2}$ or $\mathbf{j}=\mathbf{3} / \mathbf{2}$. We have already studied the first possibility. For the second $j$ value, the Hamiltonian $\hat{\mathbf{H}}^{(1)}$ is a $4 \times 4$ matrix that applying to 
the eigenstate equation (2.12), give us the matrix equation (2.17) with

$$
\hat{\mathbf{h}}=\left[\begin{array}{cccr}
\mathrm{h}_{11} & \mathrm{~h}_{12} & 0 & 0 \\
\mathrm{~h}_{12} & \mathrm{~h}_{22} & \mathrm{~h}_{23} & 0 \\
0 & \mathrm{~h}_{23} & \mathrm{~h}_{33} & \mathrm{~h}_{34} \\
0 & 0 & \mathrm{~h}_{34} & \mathrm{~h}_{44}
\end{array}\right]
$$

being the $\mathrm{h}_{i k}$ coefficients given by Eq. (2.16). Then the system of equations (2.15) becomes

$$
\left\{\begin{array}{l}
\mathrm{h}_{11} C_{n}+\mathrm{h}_{12} C_{n+m}=\mathcal{E}_{n m} C_{n} \\
\mathrm{~h}_{12} C_{n}+\mathrm{h}_{22} C_{n+m}+\mathrm{h}_{23} C_{n+2 m}=\mathcal{E}_{n m} C_{n+m} \\
\mathrm{~h}_{23} C_{n+m}+\mathrm{h}_{33} C_{n+2 m}+\mathrm{h}_{34} C_{n+3 m}=\mathcal{E}_{n m} C_{n+2 m} \\
\mathrm{~h}_{34} C_{n+2 m}+\mathrm{h}_{44} C_{n+3 m}=\mathcal{E}_{n m} C_{n+3 m}
\end{array}\right.
$$

and Eq. (2.19) is an algebraic quartic equation with coefficients

$$
\left\{\begin{array}{l}
D_{3}=-\left(\mathrm{h}_{11}+\mathrm{h}_{22}+\mathrm{h}_{33}+\mathrm{h}_{44}\right) \\
D_{2}=\mathrm{h}_{11} \mathrm{~h}_{22}+\mathrm{h}_{11} \mathrm{~h}_{33}+\mathrm{h}_{11} \mathrm{~h}_{44}+\mathrm{h}_{22} \mathrm{~h}_{33}+\mathrm{h}_{22} \mathrm{~h}_{44}+\mathrm{h}_{33} \mathrm{~h}_{44}-\left(\mathrm{h}_{12}^{2}+\mathrm{h}_{23}^{2}+\mathrm{h}_{34}^{2}\right) \\
D_{1}=-\left(\mathrm{h}_{11} \mathrm{~h}_{22} \mathrm{~h}_{33}+\mathrm{h}_{11} \mathrm{~h}_{22} \mathrm{~h}_{44}+\mathrm{h}_{11} \mathrm{~h}_{33} \mathrm{~h}_{44}+\mathrm{h}_{22} \mathrm{~h}_{33} \mathrm{~h}_{44}\right) \\
\quad+\left(\mathrm{h}_{33}+\mathrm{h}_{44}\right) \mathrm{h}_{12}^{2}+\left(\mathrm{h}_{11}+\mathrm{h}_{44}\right) \mathrm{h}_{23}^{2}+\left(\mathrm{h}_{11}+\mathrm{h}_{22}\right) \mathrm{h}_{34}^{2} \\
D_{0}=\mathrm{h}_{11} \mathrm{~h}_{22} \mathrm{~h}_{33} \mathrm{~h}_{44}-\left(\mathrm{h}_{33} \mathrm{~h}_{44} \mathrm{~h}_{12}^{2}+\mathrm{h}_{11} \mathrm{~h}_{44} \mathrm{~h}_{23}^{2}+\mathrm{h}_{11} \mathrm{~h}_{22} \mathrm{~h}_{34}^{2}-\mathrm{h}_{12}^{2} \mathrm{~h}_{34}^{2}\right),
\end{array}\right.
$$

and the eigenvalues $E_{n m}^{(1,2,3,4)}$ can be obtained by its roots, as shown in Appendix II. From Eqs. (6.8), (4.11), (2.20) and the eigenstate normalization condition we can determine the $C$ coefficients and show that the eigenstates for the total Hamiltonian can be written as

$$
\left|\Psi_{n m}^{(k)}\right\rangle=\frac{1}{\sqrt{1+\left(\gamma_{n}^{(k)}\right)^{2}+\left(\gamma_{n+m}^{(k)}\right)^{2}+\left(\gamma_{n+3 m}^{(k)}\right)^{2}}}\left[\begin{array}{c}
\gamma_{n}^{(k)}|n\rangle \\
\gamma_{n+m}^{(k)}|n+m\rangle \\
|n+2 m\rangle \\
\gamma_{n+3 m}^{(k)}|n+3 m\rangle
\end{array}\right]
$$

where

$$
\gamma_{n}^{(k)}=\Gamma_{1}^{(k)} \gamma_{n+m}^{(k)}, \quad \gamma_{n+m}^{(k)}=\frac{\Gamma_{3}^{(k)}}{1-\Gamma_{1}^{(k)} \Gamma_{2}^{(k)}}, \quad \gamma_{n+3 m}^{(k)}=\Gamma_{4}^{(k)}
$$

with $k=1,2,3,4$ and

$$
\Gamma_{1}^{(k)}=\frac{\mathrm{h}_{12}}{\mathcal{E}_{n m}^{(k)}-\mathrm{h}_{11}}, \quad \Gamma_{2}^{(k)}=\frac{\mathrm{h}_{12}}{\mathcal{E}_{n m}^{(k)}-\mathrm{h}_{22}}, \quad \Gamma_{3}^{(k)}=\frac{\mathrm{h}_{23}}{\mathcal{E}_{n m}^{(k)}-\mathrm{h}_{22}}, \quad \Gamma_{4}^{(k)}=\frac{\mathrm{h}_{12}}{\mathcal{E}_{n m}^{(k)}-\mathrm{h}_{44}},
$$

and its associated eigenvalues can be written as

$$
E_{n m}^{(k)}=\left(n+\frac{3}{2} m\right) \hbar \omega+\mathcal{E}_{n m}^{(k)}
$$

Again, the results for the intensity-dependent interaction are obtained with the change $\mathrm{h}_{i k} \rightarrow \tilde{\mathrm{h}}_{i k}$, with $i \neq k$. Basically this change implies in a modification in the $D_{0}, D_{1}$ and $D_{2}$ coefficients of the algebraic quartic equation for the eigenvalues and in the $\Gamma$-terms for the eigenstates.

For this value of $j$, the possible $n$ negative values are $r=-2 j+2=-1, r=-2 j+1=-2$ and $r=-2 j=-3$, the last one being the ground state of the system. First, using Eqs. (2.21) and (4.10) for $r=-1$, we find the following system of equations

$$
\left\{\begin{array}{l}
-\frac{1}{2} \hbar \Delta_{m} C_{0}+2 \hbar \Omega \sqrt{m !} C_{m}=\mathcal{E}_{-1 m} C_{0} \\
2 \hbar \Omega \sqrt{m !} C_{0}+\hbar\left[\Theta m(m-1)+\frac{1}{2} \Delta_{m}\right] C_{m}+\hbar \Omega \sqrt{\frac{3(2 m) !}{m !}} C_{2 m}=\mathcal{E}_{-1 m} C_{m} \\
\hbar \Omega \sqrt{\frac{3(2 m) !}{m !}} C_{m}+\hbar\left[2 \Theta m(2 m-1)+\frac{3}{2} \Delta_{m}\right] C_{2 m}=\mathcal{E}_{-1 m} C_{2 m},
\end{array}\right.
$$

and from Eq. (2.19) we have an algebraic cubic equation with coefficients 


$$
\left\{\begin{aligned}
D_{2}= & -\hbar\left[\Theta m(5 m-3)+\frac{3}{2} \Delta_{m}\right] \\
D_{1}= & \hbar^{2}\left\{\Theta m(m-1)\left[2 \Theta m(2 m-1)+\Delta_{m}\right]-\frac{1}{4} \Delta_{m}^{2}-\Omega^{2}[4 m !+3(2 m) ! / m !]\right\} \\
D_{0}= & \hbar^{3}\left\{4 \Omega^{2} m !+\frac{1}{2} \Delta_{m}\left[\Theta m(m-1)+\frac{1}{2} \Delta_{m}\right]\right\}\left[2 \Theta m(2 m-1)+\frac{3}{2} \Delta_{m}\right] \\
& \quad-\frac{3}{2} \hbar^{3} \Omega^{2} \Delta_{m}(2 m) ! / m !,
\end{aligned}\right.
$$

for the eigenvalues $\mathcal{E}_{-1 m}^{(1,2,3)}$, that can be obtained by using the recipe presented in Appendix I. From Eqs. (4.17), the normalization condition and with the eigenvalues $\mathcal{E}_{-1 m}^{(1,2,3)}$ we can calculate the $C$ coefficients and show that the eigenstates for the total Hamiltonian $\hat{\mathbf{H}}$ in this case can be written as

$$
\left|\Psi_{-1 m}^{(k)}\right\rangle=\frac{1}{\sqrt{1+\left(\gamma_{0}^{(k)}\right)^{2}+\left(\gamma_{2 m}^{(k)}\right)^{2}}}\left[\begin{array}{c}
0 \\
\gamma_{0}^{(k)}|0\rangle \\
|m\rangle \\
\gamma_{2 m}^{(k)}|2 m\rangle
\end{array}\right]
$$

where

$$
\gamma_{0}^{(k)}=\frac{2 \hbar \Omega \sqrt{m !}}{\mathcal{E}_{-1 m}^{(k)}+\frac{1}{2} \hbar \Delta_{m}}, \quad \gamma_{2 m}^{(k)}=\frac{\hbar \Omega \sqrt{3(2 m) ! / m !}}{\mathcal{E}_{-1 m}^{(k)}-\hbar\left[2 \Theta m(2 m-1)+\frac{3}{2} \Delta_{m}\right]}
$$

with $k=1,2,3$ and the eigenvalues given by

$$
E_{-1 m}^{(k)}=\frac{1}{2}(3 m-2) \hbar \omega+\mathcal{E}_{-1 m}^{(k)}
$$

The modifications required to get the results for intensity dependent interaction case can be reduced to the change of the factors $\sqrt{m !}$ by $\sqrt{m m !}$ and $\sqrt{3(2 m) ! / m !}$ by $\sqrt{6 m(2 m) ! / m !}$ in Eqs. (4.17), (4.18) and (4.20). For the case of $r=-2$, after taking the same procedure used before, we find the following system of equations

$$
\left\{\begin{array}{l}
\frac{1}{2} \hbar \Delta_{m} C_{0}+\hbar \Omega \sqrt{3 m !} C_{m}=\mathcal{E}_{-2 m} C_{0} \\
\hbar \Omega \sqrt{3 m !} C_{0}+\hbar\left[\Theta m(m-1)+\frac{3}{2} \Delta\right] C_{m}=\mathcal{E}_{-2 m} C_{m}
\end{array}\right.
$$

giving for the coefficients of the algebraic quadratic equation for the eigenvalues (2.19)

$$
\left\{\begin{array}{l}
D_{1}=-\hbar\left[\Theta m(m-1)+2 \Delta_{m}\right] \\
D_{0}=\frac{1}{2} \hbar^{2} \Delta_{m}\left[\Theta m(m-1)+\frac{3}{2} \Delta_{m}\right]-3 \hbar^{2} \Omega^{2} m ! .
\end{array}\right.
$$

So, we find

$$
\mathcal{E}_{-2 m}^{( \pm)}=\frac{\hbar}{2}\left\{\left[\Theta m(m-1)+2 \Delta_{m}\right] \pm \sqrt{\left[\Theta m(m-1)+\Delta_{m}\right]^{2}+12 \Omega^{2} m !}\right\} .
$$

Again, from Eqs. (4.24), (4.22) and the eigenstates normalization condition we can determine the $C$ coefficients and show that the eigenstates for the total Hamiltonian $\hat{\mathbf{H}}$ for this case can be written as

$$
\left|\Psi_{-2 m}^{( \pm)}\right\rangle=\frac{1}{\sqrt{1+\left(\gamma_{m}^{( \pm)}\right)^{2}}}\left[\begin{array}{c}
0 \\
0 \\
|0\rangle \\
\pm \gamma_{m}^{( \pm)}|m\rangle
\end{array}\right]
$$

where

$$
\gamma_{m}^{( \pm)}=\frac{1}{\sqrt{1+\delta_{m}^{2}} \mp \delta_{m}}
$$

and

$$
\delta_{m}=\frac{\Theta m(m-1)+\Delta_{m}}{2 \sqrt{3 m !} \Omega},
$$

being its eigenvalues given by

$$
E_{-2 m}^{( \pm)}=\frac{1}{2}(3 m-4) \hbar \omega+\mathcal{E}_{-2 m}^{( \pm)}
$$

Now, the results for the intensity dependent interaction case can be obtained with the change of the factor $\sqrt{3 m !}$ by $\sqrt{3 m m !}$ in Eqs. (4.22), (4.23), (4.24) and (4.26). Finally, using Eqs. (2.21), (4.10) and (4.11) for $r=-3$, we find

$$
E_{-3 m}=\frac{1}{2}(3 m-6) \hbar \omega+\mathcal{E}_{-3 m}
$$


with

$$
\mathcal{E}_{-3 m}=\frac{3}{2} \hbar \Delta_{m}
$$

and

$$
\left|\Psi_{-3 m}\right\rangle=\left[\begin{array}{c}
0 \\
0 \\
0 \\
|0\rangle
\end{array}\right] .
$$

For this last case we do not observe the effects of the intensity dependent interaction on the results for the eigenstates and eigenvalues of the total Hamiltonian.

After these applications we emphasize that is just a matter of simple numerical work to find the eigenstates and the energy spectra, for any higher value of $j$. It is straightforward to do an analytical calculation up to five particle system, $j_{\max }=5 / 2$, when we have a resonant situation $\left(\Delta_{m}=0\right)$ and we do not consider the presence of the nonlinearity term $(\Theta=0)$.

\section{Numerical results and discussion}

In this section we show some examples of exact numerical applications of the formalism developed in the previous sections.

In tables 1 and 2 we show the results for energy eigenvalues, $E_{n m}$, for the cases of one-particle and three-particle systems; $j=1 / 2$ and $j=1 / 2,3 / 2$, respectively. The energies $E_{n m}$ are given in units of $\hbar \omega$. The used values of the constants $\Omega, \Theta$ and $\Delta_{m}$ are chosen in a such way that the model approximation is preserved. The bosonic quantum number, $n$, is chosen as $-2 j \leq n \leq 2$ and number of photons taking part in each process as $m=1,2,3$. Results in bold face stand for the intensity-dependent interaction case, otherwise for non intensity-dependent one. As shown in the tables, for all values of $m$, the number of levels increases from 1 to $2 j+1$, as $n$ goes from $-2 j$ to $n=2$. Since the ground-state is a photon free state, the results for the intensity-dependent and non intensity-dependent cases for the ground states are equal for each value of $m$. For each value of $j$, the first excited state for $m=1$ (labeled by $n=-2 j+1$ ), also has a same value for the intensitydependent and non intensity-dependent situations. However, now this is because of the dependence on $n$ and $m$ shown by the eigenvalues, $\mathcal{E}_{n m}$. For each value of $j$, as $m$ increases the splitting among energy levels also increases. This effect is stressed in the case of intensity-dependent interaction.

Figure (1a) shows the behavior of the energy level diagram of the system for $m=1$ and $j=1 / 2$ with the energies given in units of $\hbar \omega$. In this figure we can see the split of the energy levels when we gradually turn on each component of the total Hamiltonian $\hat{\mathbf{H}}$, process that is represented by the columns (A), (B), (C), and (D). The column (A) shows only the contribution of the radiation for the energy of the system since that the other components of the total Hamiltonian are turned off. The column (B) shows the symmetric splitting in the original energy levels caused by the inclusion of the matter-radiation interaction with a strength parameter $\Omega=0.3 \omega$. The column (C) shows the effects of the inclusion of the nonlinear term with a strength parameter $\Theta=0.2 \omega$ in the total Hamiltonian. We can see that the net effect in this case is a shifting up and the increasing of the energy gap between the primary shifted levels when $n \geq-2 j+2$. Finally, the last column (D) presents the increasing of the energy gap between the primary shifted energy levels when we turn on the detuning of the system with $\Delta_{1}=0.2 \omega$. Note that the ground state energy is only modified by the presence of the nonresonant term, $\hbar \Delta_{1} \hat{\mathbf{J}}_{3}$, in the total Hamiltonian. This is because the ground state is a no photon state. It is important to note that for $j=1 / 2$ case the introduction of the nonlinear term in the Hamiltonian does not affect the results for $n$ negative values. This is because this nonlinear term takes into account a change of two photons and, thus, it is necessary to have eigenstates corresponding to bosonic states components $|k\rangle$ with $k>2$. Only for higher $j$ values we can observe the effects of the nonlinearity on the eigenstates and eigenvalues for $n$ negatives values. Figure (1b) is the intensity-dependent radiation version of Fig. (1a). If we compare these figures we conclude that the action of the intensity-dependence in the matter-radiation interaction is to generate an amplification in the energy gap of the levels which are sensitive to this dependence $(n \geq-2 j+2)$.

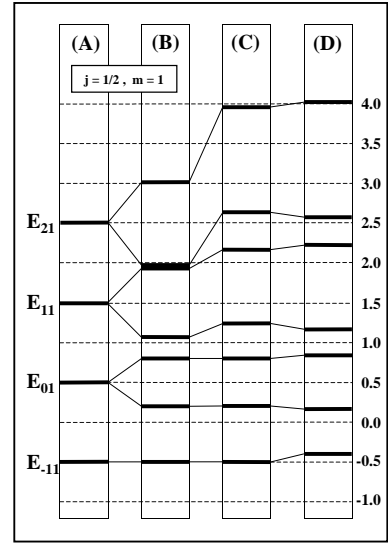

(1a)

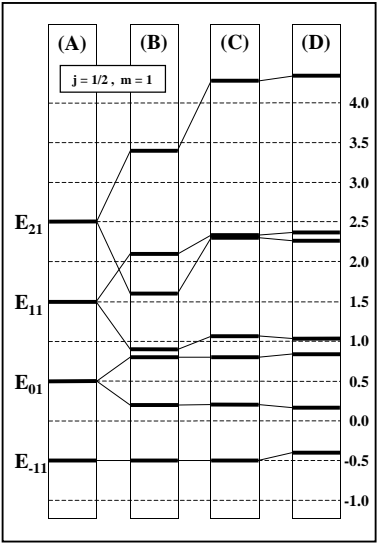

(1b)
Figure 1a. The behavior of the energy level diagram of the system for $j=1 / 2$ with the energies given in units of $\hbar \omega$. We can follow the split of the energy levels when we gradually turn on each component of the total Hamiltonian $\hat{\mathbf{H}}$, a process that is represented by columns (A), (B), (C) and (D). The set of strength used is given in the text. Figure (1b) is the the radiation intensity-dependent interaction version of Figure (1a).

Figures (2a) and (2b) are the version of Figs. (1a) and (1b) for $m=1$ and $j=3 / 2$, recpectively. We observe the same behavior presented for $j=1 / 2$ case in the splitting of the energy levels when we turn on each component of the Hamiltonian, except for the splited energy levels number and the increasing in the energy gap between the levels. 
Table 1. Values of $E_{n m}$ for $j=1 / 2$ (single particle and three particle systems).

\begin{tabular}{|c|c|c|c|c|c|c|}
\hline \multicolumn{2}{|c|}{$\overline{E_{n m}}(\hbar \omega)}$, & $j=1 /$ & $\Omega=$ & $3 \omega$ & $\Theta=\Delta_{i}$ & $=0.2 \omega$ \\
\hline \multirow{3}{*}{$n$} & \multicolumn{6}{|c|}{$m$} \\
\hline & \multicolumn{2}{|c|}{1} & \multicolumn{2}{|c|}{2} & \multicolumn{2}{|c|}{3} \\
\hline & -0.4 & -0.4 & 0.6 & 0.6 & 1.6 & 1.6 \\
\hline \multirow[t]{2}{*}{0} & 0.184 & 0.184 & 0.680 & 0.530 & 1.085 & 0.647 \\
\hline & 0.816 & 0.816 & 1.720 & 1.871 & 3.115 & 3.553 \\
\hline \multirow[t]{2}{*}{1} & 1.180 & 1.029 & 1.585 & 1.147 & 1.738 & 0.486 \\
\hline & 2.220 & 2.371 & 3.615 & 4.053 & 5.662 & 6.914 \\
\hline \multirow[t]{2}{*}{2} & 2.579 & 2.270 & 2.887 & 2.048 & 2.698 & 0.167 \\
\hline & 4.021 & 4.330 & 5.913 & 6.752 & 8.702 & 11.233 \\
\hline
\end{tabular}

Table 2. Values of $E_{n m}$ for $j=3 / 2$ (three-particle system).

\begin{tabular}{|c|c|c|c|c|c|c|}
\hline \multicolumn{2}{|c|}{$E_{n m}(\hbar \omega)}$, & \multirow{2}{*}{$j=3 / 2$} & \multirow{2}{*}{\multicolumn{2}{|c|}{$\frac{\Omega=0.3 \omega}{m}$}} & \multicolumn{2}{|c|}{$\Theta=\Delta_{m}=0.2 \omega$. } \\
\hline & \multicolumn{3}{|c|}{$m$} & & & \\
\hline \multirow[b]{2}{*}{-3} & \multicolumn{2}{|c|}{1} & \multicolumn{2}{|c|}{2} & \multicolumn{2}{|c|}{3} \\
\hline & -1.2 & -1.2 & 0.3 & 0.3 & 1.8 & 1.8 \\
\hline \multirow[t]{2}{*}{-2} & -0.829 & -0.829 & 0.606 & 0.318 & 1.847 & 0.987 \\
\hline & 0.229 & 0.229 & 2.194 & 2.482 & 4.753 & 5.613 \\
\hline \multirow[t]{3}{*}{-1} & -0.287 & -0.465 & 0.914 & -0.554 & 0.544 & -7.227 \\
\hline & 0.703 & 0.6 & 2.430 & 2.196 & 3.875 & 3.614 \\
\hline & 1.784 & 2.065 & 5.756 & 7.458 & 13.581 & 21.613 \\
\hline \multirow[t]{4}{*}{0} & 0.453 & -0.014 & 1.443 & -2.101 & -1.864 & -25.329 \\
\hline & 1.291 & 1.126 & 2.892 & 2.485 & 4.113 & 3.378 \\
\hline & 2.227 & 2.156 & 5.508 & 5.217 & 9.352 & 8.863 \\
\hline & 3.629 & 4.331 & 10.957 & 15.200 & 27.999 & 52.688 \\
\hline \multirow[t]{4}{*}{1} & 1.496 & 0.642 & 2.351 & -2.457 & -2.012 & -33.215 \\
\hline & 2.628 & 2.236 & 4.388 & 3.287 & 5.081 & 2.914 \\
\hline & 4.031 & 4.116 & 8.203 & 8.205 & 13.043 & 13.641 \\
\hline & 5.846 & 7.006 & 14.658 & 20.565 & 34.688 & 67.459 \\
\hline \multirow[t]{4}{*}{2} & 2.912 & 1.620 & 3.538 & -2.811 & -2.147 & -42.408 \\
\hline & 4.405 & 3.819 & 6.405 & 4.496 & 6.508 & 2.197 \\
\hline & 6.230 & 6.477 & 11.299 & 11.740 & 17.264 & 19.623 \\
\hline & 8.454 & 10.083 & 18.758 & 26.576 & 41.975 & 84.188 \\
\hline
\end{tabular}

Figure (3a) shows the behavior of the energy level diagram of the system for $j=3 / 2, n=2$ and $m=1,2,3$; with energies given in units of $\hbar \omega$. In this Figure we can see the split of the energy levels when we gradually turn on each component of the total Hamiltonian $\hat{\mathbf{H}}$, process that is represented by columns (A), (B), (C) and (D). The leftmost column (A) shows only the contribution of the radiation for the energy of the system since the other components of the total Hamiltonian are turned off. The next column (B) shows the symmetric splitting in the original energy levels caused by the inclusion of the matter-radiation interaction with a strength parameter $\Omega=0.3 \omega$. The third column (C) shows the effects of the inclusion of the nonlinear term with a strength parameter $\Theta=0.2 \omega$ in the total Hamiltonian.
We can see that the net effect in this case is a shifting up and the increasing of the energy gap between the primary shifted levels. The last column (D) presents the increasing of the energy gap between the primary shifted energy levels when we turn on the detuning of the system with $\Delta_{m}=0.2 \omega$. Finally, we note that as $m$ increases, the gap between levels also increases. This kind of behavior of the energy levels is present for all excited states.

Figure (3b) is the intensity-dependent version of Fig. (3a). If we compare these two set of figures we conclude that the action of the intensity-dependence in the matterradiation interaction is to generate an amplification in the energy gap of the levels. 


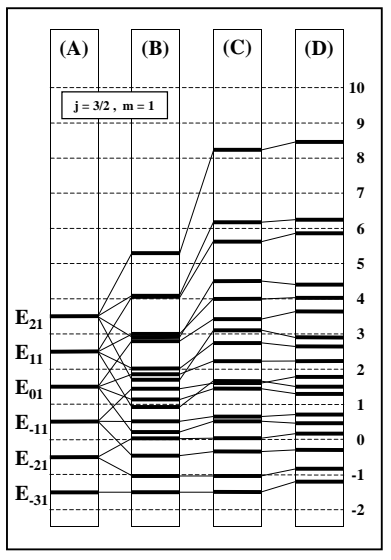

(2a)

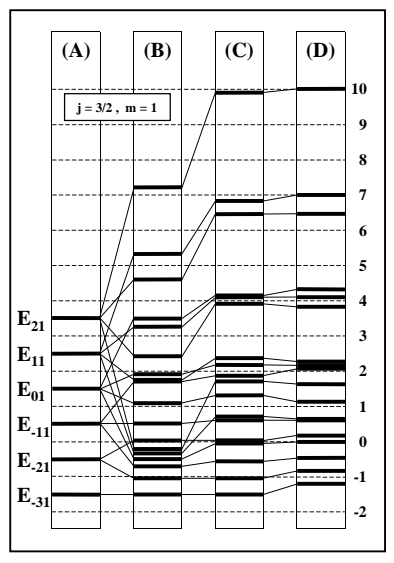

(2b)
Figure 2a. The $j=3 / 2$ version of Figs. (1a) and (1b).

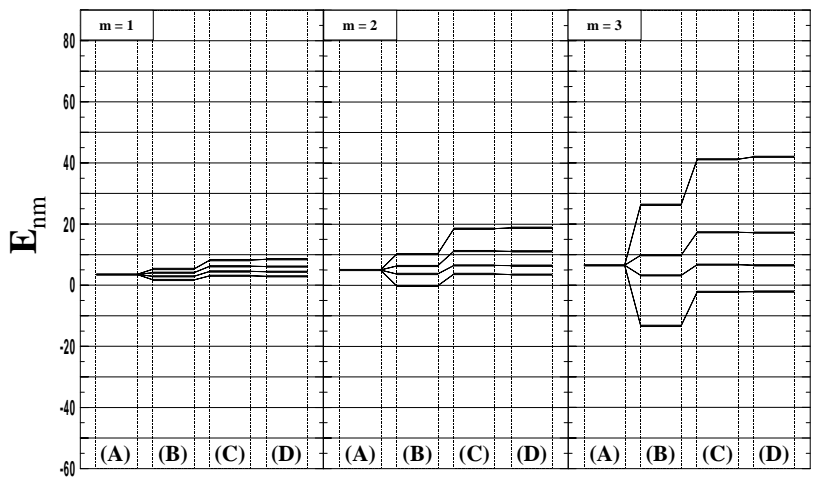

(3a)

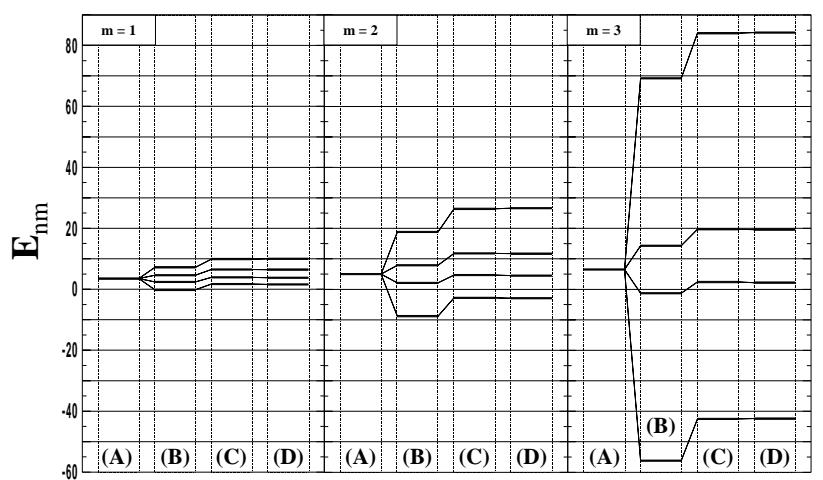

(3b)

Figure 3a. Some of energy levels for a $j=3 / 2$ system with $n=2$ and $m=1,2$ and 3 , as each component of the total Hamiltonian $\hat{\mathbf{H}}$ is progressively added, a process that is represented by columns (A), (B), (C), and (D). Figure (3b) is the radiation intensity-dependent version of Figure (3a).
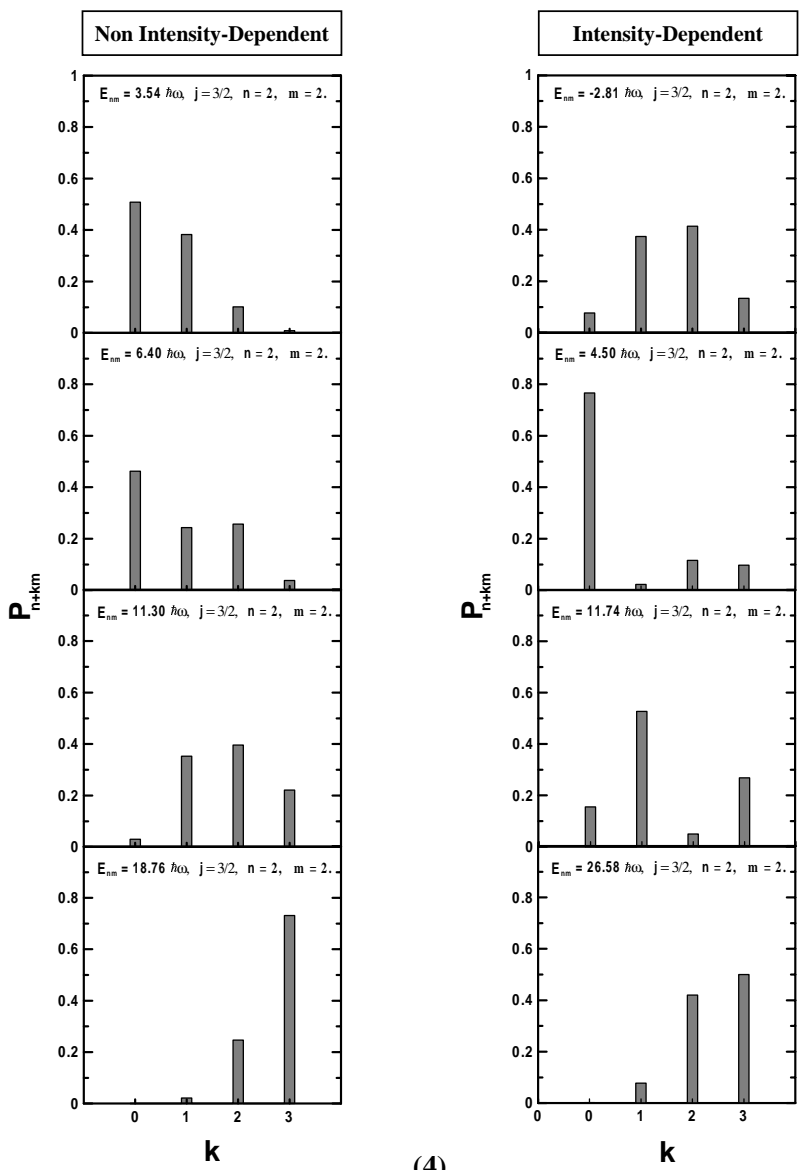

Figure 4. Weight of each component, $P_{n+k m}=C_{n+k m}^{2}$, in the composition of the state vectors, $\left|\Psi_{n m}^{(1,2,3,4)}\right\rangle$ for $j=3 / 2$, and $n=m=2$. The left column is for a non intensity-dependent interaction and the right column is for an intensity-dependent one.

Figure (4) shows the weight of each component, $P_{n+k m}=C_{n+k m}^{2}$, with $k=0,1,2$ and 3 , in the composition of the state vectors, $\left|\Psi_{n m}^{(1,2,3,4)}\right\rangle$ for $j=3 / 2$, and $n=m=2$. The values of parameters $\Omega, \Theta$ and $\Delta_{m}$ are the same as in the tables 1 and 2. In this Figure are shown the $2 j+1$ states, from the less energetic state in the top to the more one in the bottom and the size of the vertical bars are proportional to the weight of the correspondent component. The column to the left is for the non intensity-dependent situation and that one to the right is for the intensity-dependent case. Clearly, in the non intensity-dependent situation and for the lowest energy eigenvalue (in the top), the components more important in the composition of the eigenstate correspond to the lower bosonic states. As the energy eigenvalue increases (going down in the figure), this behavior gradually change, in a such way that for the highest energy eigenvalue (in the bottom) we observe that the components with more importance in the composition of the eigenstate correspond to the higher bosonic states. For the intensitydependent situation and for the positive energy eigenvalues, we observe the same behavior as describe above for the relative importance of the components in the composition of the eigenstate. However, for the only one eigenstate with a negative energy eigenvalue (in the top), we see that interme- 
diate components present a higher relative importance than the components in the extremes. A non observed vertical bar in the figures does not mean that the respective component is null, but only that in the figure scale its relative value can not be represented.

Figure (5) is the same as for Figure (4) with $n=m=3$. As we can see, for negative (top of each column) and positive energy eigenvalues, the general behavior for the relative importance of the components is the same as describe before.

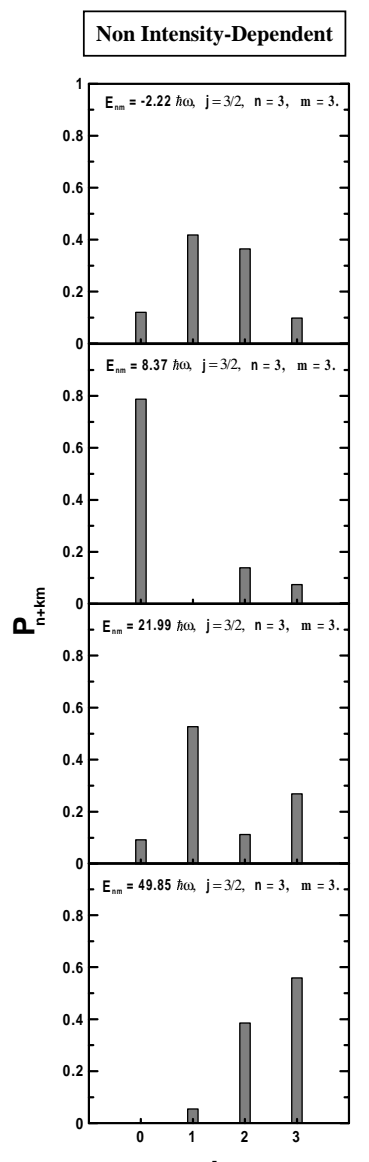

(5)

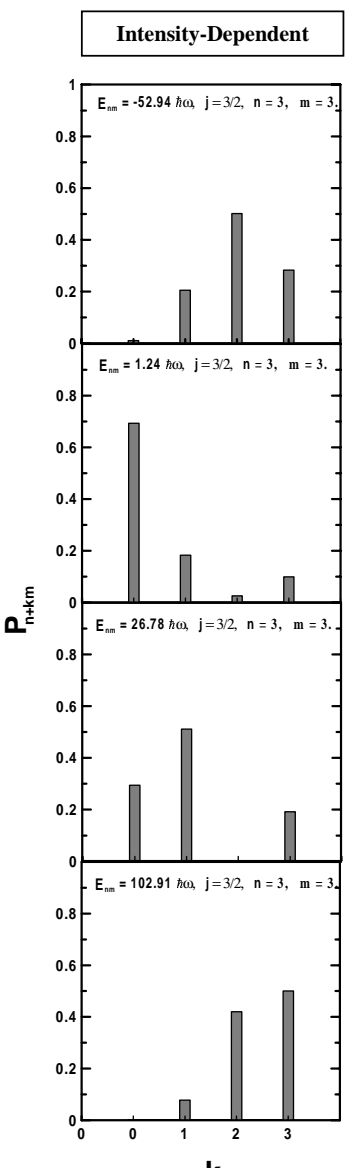

Figure 5. The version of Fig. (4) for $n=m=3$.

\section{Conclusions}

In this article we introduced a model for interaction between the matter, consisting of $N$ two-level particles, and the radiation via an $m$-photon process, with $m=1,2,3, \ldots$ The model includes the nonlinear effects related with the nonlinear polarisability in a nonlinear medium and nonlinear effects due an intensity-dependent radiation coupling. We present the exact results for the eigenstates and eigenvalues for some different values of $j$ in terms of the photon number $m$. We compare the different spectra to evaluate the effects of the photon number involved in the interaction and the different sources of nonlinearity and also discuss some aspects of the composition of the eigenstates and of the spectra obtained in these cases.

We emphasize that our general model makes possible to resolve simultaneously problems with different Hamiltonians; as we set $\Theta=\Delta_{m}=0$, our model reduces to a bare resonant model for a multi-atom system interacting with an electromagnetic radiation via a multi-photon process; if we put $\Theta=0, \Delta_{m} \neq 0$, we get a bare multi-atom -photon non resonant model and if we set $\Theta \neq 0, \Delta_{m}=0$, we have a multi-atom -photon resonant model with a nonlinearity. We showed that it is straightforward to generalize all these situations for an intensity-dependent interaction.

A related quantum mechanical model for the propagation of light through a nonlinear medium taking into account the nonlinear polarisability of radiation was presented in Ref. [15]. Our Hamiltonian can be considered a generalization of this model. Indeed, for $m=1$, using the Holstein-Primakoff boson mapping of the angular momentum algebra:

$$
\left\{\begin{array}{l}
\hat{\mathbf{J}}_{+}=\sqrt{2 j} \hat{b}^{\dagger}\left(\sqrt{1-\frac{1}{2 j} \hat{b}^{\dagger} \hat{b}}\right) \\
\hat{\mathbf{J}}_{-}=\sqrt{2 j}\left(\sqrt{1-\frac{1}{2 j} \hat{b}^{\dagger} \hat{b}}\right) \hat{b} \\
\hat{\mathbf{J}}_{3}=-1+\hat{b}^{\dagger} \hat{b}
\end{array}\right.
$$

and introducing the scaled matter-radiation interaction parameter

$$
\Omega=\frac{\Omega^{\prime}}{\sqrt{2 j}}
$$

one sees that in the limit $j \rightarrow \infty$, substitution of Eq. (6.1) into Eqs. (2.1), (2.1), (2.2) yields the Hamiltonian

$$
\hat{\mathbf{H}}=\hbar \omega \hat{a}^{\dagger} \hat{a}+\hbar \omega_{0} \hat{b}^{\dagger} \hat{b}+\hbar \Omega^{\prime}\left(\hat{a} \hat{b}^{\dagger}+\hat{a}^{\dagger} \hat{b}\right)+\hbar \Theta \hat{a}^{\dagger 2} \hat{a}^{2}
$$

up to a constant. Eq. (6.3) is the Hamiltonian studied in Ref. [15]. Our Hamiltonian can also be considered as an exactly-solvable coupled-channels problem. As many interesting problems in areas ranging from nuclear physics [16] to the study of transitions from metastable states [17] have coupled-channels form, our Hamiltonian can be a starting point for other models.

\section{Appendix I}

The roots of the cubic algebraic equation 


$$
E_{q n}^{3}+D_{2} E_{q n}^{2}+D_{1} E_{q n}+D_{0}=0
$$

can be obtained by[18]

$$
\left\{\begin{array}{l}
E_{q n}^{(1)}=\left(E_{+}+E_{-}\right)-\frac{1}{3} D_{2} \\
E_{q n}^{(2)}=-\frac{1}{2}\left(E_{+}+E_{-}\right)-\frac{1}{3} D_{2}+\frac{i \sqrt{3}}{2}\left(E_{+}-E_{-}\right) \\
E_{q n}^{(3)}=-\frac{1}{2}\left(E_{+}+E_{-}\right)-\frac{1}{3} D_{2}-\frac{i \sqrt{3}}{2}\left(E_{+}-E_{-}\right) .
\end{array}\right.
$$

where $E_{+}$and $E_{-}$, that must be complex conjugate, are given by

$$
E_{ \pm}=\left[u \pm \sqrt{p^{3}+u^{2}}\right]^{1 / 3}
$$

with

$$
\left\{\begin{array}{l}
p=\frac{1}{9}\left(3 D_{1}-D_{2}^{2}\right) \\
u=\frac{1}{6}\left(D_{1} D_{2}-3 D_{0}\right)-\frac{1}{27} D_{2}^{3}
\end{array}\right.
$$

\section{Appendix II}

The roots of the quartic algebraic equation

$$
E_{q n}^{4}+D_{3} E_{q n}^{3}+D_{2} E_{q n}^{2}+D_{1} E_{q n}+D_{0}=0
$$

can be obtained by[19]

$$
\left\{\begin{array}{l}
E_{q n}^{(1,2)}=-\frac{1}{4}\left(D_{3}+2 B_{a}\right) \pm \sqrt{\frac{1}{16}\left(D_{3}+2 B_{a}\right)^{2}-\left(B_{k}+B_{b}\right)} \\
E_{q n}^{(3,4)}=-\frac{1}{4}\left(D_{3}-2 B_{a}\right) \pm \sqrt{\frac{1}{16}\left(D_{3}-2 B_{a}\right)^{2}-\left(B_{k}-B_{b}\right)}
\end{array}\right.
$$

where

$$
\left\{\begin{array}{l}
B_{a}=\sqrt{2 B_{k}-D_{2}+\frac{1}{4} D_{3}^{2}} \\
B_{b}=\left(B_{k} D_{3}-D_{1}\right) /\left(2 B_{a}\right) \\
B_{k}=B_{+}+B_{-}+\frac{1}{6} D_{2},
\end{array} \quad \text { with } \quad B_{ \pm}=\left[u \pm \sqrt{u^{2}+p^{3}}\right]^{1 / 3},\right.
$$

and

$$
\left\{\begin{array}{l}
p=\frac{1}{36}\left[3\left(D_{1} D_{3}-4 D_{0}\right)-D_{2}^{2}\right] \\
u=\frac{1}{216} D_{2}^{3}-\frac{1}{48}\left[D_{1}\left(D_{2} D_{3}-3 D_{1}\right)+D_{0}\left(8 D_{2}-3 D_{3}^{2}\right)\right] .
\end{array}\right.
$$

\section{Acknowledgments}

This work was supported in part by the U.S. National Science Foundation Grants No. INT-0070889 and PHY0070161 at the University of Wisconsin, and in part by the University of Wisconsin Research Committee with funds granted by the Wisconsin Alumni Research Foundation. M.A.C.R. acknowledges the support of Fundação de Amparo à Pesquisa do Estado de São Paulo - FAPESP (Contract No. 98/13722-2). A.N.F.A. and M.A.C.R. acknowledge the support of Conselho Nacional de Pesquisa (CNPq) (Contract No. 910040/99-0). A.N.F.A. and M.A.C.R. thank to the Nuclear Theory Group at University of Wisconsin for their very kind hospitality.

\section{References}

[1] E. T. Jaynes and F. W. Cummings, Proc. IEEE 51, 89 (1963).

[2] B. Buck and C. V. Sukumar, Phys. Lett. A 81, 132 (1981).

[3] C. C. Gerry, Phys. Rev. A 37, 2683 (1988).
[4] M. Chaichian, D. Ellinas, and P. Kulish, Phys. Rev. Lett. 65, 980 (1990).

[5] B. Deb and D. S. Ray, Phys. Rev. A 48, 3191 (1993).

[6] V. Buzěk, Phys. Rev. A 39, 3196 (1989).

[7] W. R. Mallory, Phys. Rev. 188, 1976 (1969); I. R. Senitzky, Phys. Rev. A 3, 421 (1971).

[8] P. D. Drummond and D. F. Walls, J. Phys. A 13, 725 (1980).

[9] A. Rybin, G. Miroshnichenko, I. Vadeiko, and J. Timonen, J. Phys. A: Math. Gen. 32, 8739 (1999).

[10] C. Flytzanis and C. L. Tang, Phys. Rev. Lett. 45, 441 (1980).

[11] N. Imoto, H. A. Haus, and Y. Yamamoto, Phys. Rev. A 32, 2287 (1985).

[12] A. N. Aleixo, A. B. Balantekin, and M. A. Cândido Ribeiro, J. Phys. A 33, 3173 (2000) [quant-ph/0001049].

[13] A. N. Aleixo, A. B. Balantekin, and M. A. Cândido Ribeiro, J. Phys. A 34, 1109 (2001) [quant-ph/0101024].

[14] W. H. Louisell, Quantum Statistical Properties of Radiation, chap. 5, J. Wiley - 1973. 
[15] G. S. Agarwal and R. R. Puri, Phys. Rev. A 39, 2969 (1989).

[16] A. B. Balantekin and N. Takigawa, Rev. Mod. Phys. 70, 77 (1998) [nucl-th/9708036].

[17] P. Hänggi, P. Talkner, and M. Borkovec, Rev. Mod. Phys. 62, 251 (1990)
[18] M. Abramowitz and I. A. Stegun, Handbook of Mathematical Functions, Dover - 1972.

[19] N. B. Conkwright, Introduction to the Theory of Equations, chap. 5, Ginn and Company - 1941. 